\title{
Germanica
}

\section{Regionalität und Modernisierung in der neuesten deutschsprachigen Kriminalliteratur (1990-2015)}

Nebst einigen Lektüreempfehlungen

Jochen Vogt

\section{OpenEdition}

\section{Journals}

Édition électronique

URL : http://journals.openedition.org/germanica/3172

DOI : 10.4000/germanica.3172

ISSN : 2107-0784

\section{Éditeur}

Université de Lille

\section{Édition imprimée}

Date de publication : 30 septembre 2016

Pagination : 13-39

ISBN : 9782913857377

ISSN : 0984-2632

\section{Référence électronique}

Jochen Vogt, «Regionalität und Modernisierung in der neuesten deutschsprachigen Kriminalliteratur (1990-2015) », Germanica [Online], 58 | 2016, Online erschienen am: 30 September 2018, abgerufen am 06 Oktober 2020. URL : http://journals.openedition.org/germanica/3172 ; DOI : https://doi.org/

10.4000/germanica.3172 
Actualité du roman policier 



\title{
Regionalität und Modernisierung in der neuesten deutschsprachigen Kriminalliteratur (1990-2015).
}

Nebst einigen Lektüreempfehlungen

\author{
Jochen VOGT \\ Professor em. \\ Universität Duisburg-Essen
}

Seit den frühen 1990er Jahren ist in der deutschsprachigen Kriminalliteratur, also im wiedervereinigten Deutschland, in Österreich und der deutschsprachigen Schweiz, ein enormer quantitativer Zuwachs der Produktion und eine unbestreitbare und genrespezifische Steigerung der literarischen Qualität zu beobachten, mit der sie endlich auch Anschluss an die internationalen Standards gefunden hat. Damit setzt eine bis heute anhaltende Ausdifferenzierung von verschiedenen Subgenres, thematischen Komplexen und individuellen Schreibweisen ein - ein Prozess, der sich allerdings, weitgehend gleichzeitig, auf verschiedenen literarischen Niveaustufen und mit unterschiedlich großem kommerziellem Erfolg entfaltet. Insofern ist die Entwicklung des Genres nicht auf einen festen Typus zu reduzieren und schwieriger zu fassen, als es noch beim sogenannten ,Soziokrimi“ der 1970er und frühen 1980er Jahre der Fall war. Im Folgenden versuche ich, einige wichtige Tendenzen unter den Stichwörtern „Regionalismus“ und „Modernisierung“ zu fassen und anschaulich zu machen - ohne die angesprochenen Unterschiede einzuebnen. 


\section{Ein Brief}

Im Jahr 2010 erhielt ich, wie andere Kritiker auch, einen Werbebrief aus dem traditionsreichen Hause S. Fischer, also dem Hausverlag von Hofmannsthal, Freud, Kafka und Thomas Mann. Darin teilte einer der heute umsatzstärksten Autoren des Hauses, Klaus-Peter Wolf, mir ,persönlich' Folgendes über sich und seine Werke mit: „Nur weil Klaus-Peter Wolfs Kriminalromane in Zeit und Raum sehr genau verortet sind und im Titel das Wort ,Ostfriesland' tragen, werden sie oft als Regionalkrimi abgetan. Wer sie aber liest, hat nie das Gefühl, einen Regio-Krimi zu lesen, sondern ein genaues Psychogramm einer Gesellschaft am Rande des Abgrunds." Ostfriesland ist eine ruhige, geographisch etwas randständige Gegend, die vor allem für ihre Teemischung, bewährte Regenkleidung, die Wortkargheit der Einheimischen und das vielleicht beste deutsche Bier bekannt ist (und natürlich auch für die „Ostfriesenwitze“, in denen die sonstigen Deutschen jene Wortkargheit bösartig als Einfalt missdeuten). Nun gibt es also auch die Ostfriesenkrimis von Klaus-Peter Wolf, insgesamt neun Titel, vom Ostfriesenkiller (2007) bis zur Ostfriesenwut (2015), in einer geschätzten Gesamtauflage von etwa 5 Millionen Exemplaren, meist im Taschenbuch. Da ist man versucht, die Werbung auf dem Einwickelpapier einer ostfriesischen Metzgerei zu übernehmen: „Regional ist erste Wahl!“ Tatsächlich nutzt der Verlag dann aber, in Verbindung mit einer lebensgroß aufgestellten Ganzkörperfotografie des Autors, in den Buchhandlungen den nicht sehr viel subtileren Slogan „Ostfriesland hat den Superstar!“”

Was sagt uns dies? Das Wortfeld „Region/regional“ hat sich längst in der deutschen Umgangssprache etabliert. Es bezeichnet nicht nur Wurstwaren und sonstige Lebensmittel, sondern auch literarische Werke, wobei es offenbar einerseits als Marken-Merkmal und Gütesiegel in Anspruch genommen, andererseits aber als negatives Wertungskriterium gefürchtet und abgewehrt wird. „Etwas ist nicht geheuer," möchte man da mit dem philosophischen Krimileser Ernst Bloch sagen, „damit fängt es an. [...] Der Fall selber muß es in sich haben, so ganz nebenbei.“"

\section{Was heißt Regionalität?}

Tatsächlich tauchen der Begriff der Region und das angelagerte Wortfeld in deutschen Wörterbüchern erst in den 1950er Jahren auf. Das legt die Vermutung nahe, dass sie traditionelle Begrifflichkeiten

1. - Ernst Bloch, „Philosophische Ansicht des Detektivromans (1965)“, in: Jochen Vogt (Hrsg.), Der Kriminalroman. Poetik - Theorie - Geschichte, München, Fink, 1998, S. 138. 
ersetzen, die durch den nationalsozialistischen Missbrauch kaum noch gebrauchsfähig, oder zumindest beschädigt sind: Heimat, Volk, Nation (von NS-Archaismen wie „Gau“ und „Lebensraum“ zu schweigen). Dies wiederum ist nicht falsch, aber bei weitem nicht hinreichend, um die Konjunktur des Regionalen zu erklären, die sich seit Mitte der 1970er Jahre eben nicht nur in der Bundesrepublik anbahnt. Sie wird, lange bevor jemand das Schlagwort Globalisierung kennt oder benutzt, aus sozialen und politischen Protest- und Separationsbewegungen mit antizentralistischer Stoßrichtung gespeist, zunächst vor allem in Frankreich und Spanien. Für Deutschland war der regionale, gleichzeitig aber staatsgrenzen- und klassenüberschreitende, und vor allem erfolgreiche Widerstand gegen den Bau eines Atomkraftwerks in Wyhl am Oberrhein ein Schlüsselereignis.

Inzwischen ist Regionalität eine in Alltagssprache und Alltagshandeln selbstverständliche Kategorie, aber längst auch ein Schlüsselwort für Maßnahmen und Strategien ,von oben“ geworden, besonders in der Verkehrs- und Infrastrukturpolitik, in der Energiewirtschaft, im Tourismus und der Image- oder Konsumwerbung. Wer heute beispielsweise in Freiburg in eine Straßenbahn steigt, tut dies am besten mit der Regio-Karte, die ihn oder sie bis an oder gar über die französische bzw. Schweizer Grenze bringt. Die Tourismusmanager arbeiten an einer einheitlichen Werbestrategie für die dreistaatliche „Regio Oberrhein“, von den Münstertürmen in Freiburg, Strasbourg und Basel begrenzt, und zielen damit auf eine neue zahlungskräftige Klientel aus Fernost oder Russland, kaum noch aus den USA, dafür auf die ,regionalen Nachbarn in Italien und Frankreich.

Der Begriff des Regionalen ist also durchaus ambivalent: Er bezeichnet einerseits den Protest oder Widerstand gegen zentralistische Planungen, gegen die Metropolen, auch gegen Globalisierung, und andererseits ein neues, heutigen Mentalitäten und Lebensgewohnheiten angepasstes Konzept der Beeinflussung und Steuerung von Bevölkerung und Konsumenten. Das reflektiert die zunehmende Angleichung von städtischen und ländlichen Lebensformen, die durch Modernisierung und Globalisierung bewirkt werden; die „Region“ ersetzt so auch die traditionelle Bezeichnung „Provinz“ und verwischt deren Gegensatz zur „Metropole“.

Dabei bleiben Begriffsdefinitionen für die Region als „Raum mittlerer Größe", wie sie in der neueren Sozialgeographie und Stadtsoziologie versucht werden, relativ allgemein und unscharf wie in der folgenden Paraphrase nach dem führenden Sozialgeographen Benno Werlen von der Universität Jena:

Regionen integrieren eine heterogene Population mit gestreuten Milieus, pluralen Lebensstilen, Migrationskulturen, Transnationalitäten 
etc. Sie können als flexible Einheiten verstanden werden, die dem Umstand Rechnung tragen, dass lokal verankerte, traditionale Lebensformen nicht mehr dominieren, sondern nur noch eine Möglichkeit neben anderen darstellen. [...] Sie kommen einem subjektiven Bedarf an Zugehörigkeit entgegen, der sich jedoch weniger am Überkommenen als an [alltäglichen] Routinen orientiert, damit aber auch die prinzipielle Austauschbarkeit der Regionen voraussetzt. ${ }^{2}$

In diesem Zusammenhang können dann kollektive Diskurse und Symbole (und damit auch populäre Formen der Unterhaltungskultur wie der Krimi als Buch, Film und im Fernsehen) funktional und rezeptionsästhetisch durchaus wichtig werden. Allerdings hat auch die neuere, kulturwissenschaftlich ,gewendete' Literatur- und Medienwissenschaft in Deutschland das analytische Potential dieser Perspektive bisher noch kaum ausgeschöpft.

\section{Einige Voraussetzungen für den Regionalkrimi}

Die Tradition einer deutschsprachigen Kriminalliteratur ist bekanntlich aus literarischen wie historischen Gründen schwach und diskontinuierlich ${ }^{3}$. Der „Neue deutsche Kriminalroman“ der frühen 1970er Jahre verdankte sich einerseits dem ,sozialliberalen“ Zeitgeist, insofern er vor allem auf soziale Ursachen von Kriminalität abhob, andererseits der sehr verzögert einsetzenden Rezeption der amerikanischen und europäischen Genre-Klassiker, auch hier im Buch, im Film und im Fernsehen. (Auch in den USA wird übrigens der Beginn einer regionalization des Genres auf die frühen 1970er Jahre datiert. Anders gesagt: Ohne TV-Serien wie Rockford P. I und The Streets of San Francisco hätte es vermutlich auch keinen deutschen Regionalkrimi gegeben.) Nach einer kurzen Phase des Experimentierens mit fiktiven Handlungsorten pegeln sich praktisch alle deutschen Autoren auf real existierende, wiedererkennbare und überprüfbare „Tatorte“ ein. Zur gleichen Zeit, genau 1970, startet die Fernsehserie Tatort, mit fast 1000 Folgen bisher die erfolgreichste deutsche Fernsehsendung, ja die wichtigste Errungenschaft

2. - Wilhelm Ammann, ,,,Regionalität' in den Kulturwissenschaften“, in: W. A., Georg Mein, Rolf Parr (Hrsg.), Periphere Zentren oder zentrale Peripherien? Kulturen und Regionen Europas zwischen Globalisierung und Regionalität, Heidelberg, Synchron, 2008, S. 13-30, Zitat S. 19; vgl. Benno Werlen, „Alltägliche Regionalisierungen unter räumlich-zeitlich entankerten Lebensbedingungen“, in: Informationen zur Raumentwicklung, H. 9/10 (2000), S. 611-622; sowie das Standardwerk von Benno Werlen, Sozialgeographie alltäglicher Regionalisierungen, 3 Bde. 1995, 2007.

3. - Detaillierter als sonst üblich wird die deutschsprachige Kriminalliteratur in der neuesten Überblicksdarstellung behandelt, die jedoch den internationalen Rahmen nicht vernachlässigt: Thomas Kniesche, Einführung in den Kriminalroman, Darmstadt, WBG, 2015. 
der westdeutschen Alltagskultur. Für unser Thema ist sie relevant, weil sowohl in ihrer Produktionsweise als auch inhaltlich ganz regionalistisch strukturiert: Die derzeit neun unabhängigen Anstalten unter dem Dach der ARD (allgemein „Das Erste Programm“ genannt) produzieren jeweils eigene (teils sogar mehrere parallele) Serien an verschiedenen Schauplätzen aus ihrem jeweiligen Sendegebiet, von Kiel bis Konstanz, von Saarbrücken bis Leipzig. Der durchschlagende Erfolg in mehr als 45 Jahren hat eine Reihe von spin-offs und Konkurrenzserien in der ARD selbst, im ZDF und bei den privaten Sendern inspiriert, die heute ein deutschlandweites und wochendeckendes Netzwerk bilden. Ganz offensichtlich spricht ein erkennbar lokaler oder regionaler Schauplatz und Handlungsrahmen die Emotionen, Erwartungen und das Orientierungsbedürfnis vieler Zuschauer an - ganz gleich ob man dies nun als kompensatorische Reaktionen auf die rasant zunehmenden Erfahrungen der Entgrenzung und virtuellen Vernetzung in einer globalisierten Welt erklären mag oder nicht.

Für die Entwicklung eines eigenständigen deutschen Kriminalromans mit analytischem Anspruch und literarischem Niveau war Tatort nicht nur wichtig, weil einige ältere Autoren, die dem „Neuen deutschen Krimi“ zuzurechnen sind, wie etwa Felix Huby (der mehr Drehbücher für diese Reihe verfasst hat als irgendein anderer), ihre Stoffe und Stories in beiden Medien verwertet haben; sie sind allerdings inzwischen von einer jüngeren, stärker fernseh- und filmästhetisch orientierten Generation abgelöst worden. Vielmehr hat diese „Reihe von Serien" unter dem Namen Tatort ${ }^{4}$ insgesamt, trotz unvermeidlich erheblicher Niveauschwankungen zwischen einzelnen Serien und Folgen, das Fernseh- und Lesepublikum, aber auch die Autoren an handwerkliche Mindeststandards gewöhnt und damit das Krimigenre in Deutschland insgesamt breitenwirksam und salonfähig gemacht. Nicht vergessen sollte man schließlich, dass auch die deutsche mainstream-Literatur, also etwa die der Autoren und Autorinnen aus der Gruppe 47, nach 1945 durchaus regionalistisch geprägt war, denken wir nur an Bölls Rheinland und Walsers Bodensee, an Grassens Danzig oder an Uwe Johnsons Mecklenburg und auch an seine Upper West Side in New York City.

4. - $\mathrm{Zu} \mathrm{den} \mathrm{einzelnen} \mathrm{Folgen} \mathrm{und} \mathrm{Serien:} \mathrm{Titel,} \mathrm{Daten} \mathrm{und} \mathrm{Fakten:}$ www.Tatort-Fundus.de - Zur Forschung: Jochen Vogt, ,Tatort - Der wahre deutsche Gesellschaftsroman. Eine Projektskizze“, in: J. V. (Hrsg.), Medien Morde. Krimis intermedial, München, Fink, 2005, S. 111-129; Christian Hißnauer, Stefan Scherer, Claudia Stockinger (Hrsg.), Föderalismus in Serie. Die Einheit der ARD-Reihe „Tatort" im historischen Verlauf, München, Fink, 2014; Hendrik Buhl, Tatort. Gesellschaftspolitische Themen in der Krimireihe, Konstanz, München, UKV, 2013. 


\section{4. ...und ein paar frühe Versuche}

Das heißt sicher nicht, dass die frühen Regional- oder Lokalkrimis sich bewusst an solchen hochliterarischen Vorbildern orientiert hätten. Doch ist aufschlussreich, wie sich der regionale Krimi als Typus Mitte der 1980er Jahre herausgebildet hat und zunehmend erfolgreich war. Das untersucht man am besten an einem - natürlich: regionalen - Beispiel.

Im Jahr 1986 gründet der kaufmännische Angestellte Friedrich Hitzbleck in Essen einen eigenen Verlag, in dem er als „Conny Lens“ einige Krimis mit komödiantischem Einschlag publiziert und unter das Seriensignet Steeler Straße stellt. Das war und ist eine enge und lebhafte, von sozialen Kontrasten und ethnischer Vielfalt geprägte Einkaufsstraße am Essener Wasserturm, der durch die blutigen Ruhrkämpfe von 1923 in die Zeitgeschichte eingegangen ist. Lens bleibt aber ganz gegenwärtig, verbindet Detailrealismus und regionalsprachliche Zitate mit slapstickElementen, publiziert später im kurzlebigen Haffmanns-Verlag, bevor er sich dauerhaft als Drehbuchschreiber für TV-Vorabendserien, öfters mit Berliner Schauplatz, verdingt. Schon 1984 hatte Corinna Kawaters, Soziologiestudentin an der Ruhr-Universität in Bochum, den schmalen, anarchistisch-feministisch eingefärbten Krimi Zora Zobel findet die Leiche herausgebracht, der im alternativen Milieu zum Kultbuch wurde, obwohl (oder weil) der Szenenjargon die dürftige Handlung kaum verdecken kann. Zora Zobel war damals nur in linken Buchläden und über den 2001-Versand erhältlich. Die Autorin hat nach vielen Jahren, die sie im südamerikanischen Untergrund verbrachte, 2010 mit ihrer Heldin Zora einen mäßig erfolgreichen Comeback-Versuch gewagt.

Biederer, aber auch solider erzählt Werner Schmitz, Kriminalbeamter aus Bochum, der 1985 mit Dienst nach Vorschu $\beta$ debütierte und bis heute ein beliebter Schulautor ist. Er nutzte (wie ansatzweise auch Kawaters) ein für die Region besonders charakteristisches Merkmal des Ruhrgebiets: seine gitterartige Topographie und Infrastruktur, die Nachbarschaft selbständiger Großstädte und besonders die Schnellstraßen, was der Erzählkonstruktion und Handlung eine eigene räumliche Dynamik verleiht. Heutige Autoren des „Ruhrgebietskrimi““ wie Jörg Juretzka oder Norbert Horst nehmen dieses Konzept auf, wie wir noch sehen werden, auch wenn sie es dann narrativ und stilistisch völlig unterschiedlich umsetzen.

Die historische Gerechtigkeit gebietet es jedoch, auf einen Sonderund Einzelfall hinzuweisen, auch wenn er unsere Chronologie in Frage stellt. Denn schon 1975 hatte Jürgen Lodemann, einfalls- und einflussreicher Literaturredakteur beim Südwestfunk in Baden-Baden, der sich als Autor bis heute in allen Genres tummelt, einen außerordentlich witzigen, im Zitieren wie im Dekonstruieren von Ruhrgebietsklischees treffsicheren Krimi über seine Heimatstadt Essen herausgebracht: Anita 
Drögemöller und Die Ruhe an der Ruhr. Hauptfiguren sind eine freischaffende Sexarbeiterin für die allerhöchsten Kreise mit goldenem Herzen und besonders lockerem Mundwerk, eben Anita, sowie der aus Ostwestfalen zugezogene Kommissar Langensiepen, der sie zuerst verdächtigt, ihr dann verfällt und sie schließlich nicht zu retten vermag. Als Folie eines pikanten Mordfalles verwendet Lodemann das in dieser Stadt bis heute spürbare topographische und soziale Gefälle zwischen Süd und Nord, Oben und Unten, das sich vor allem in den präzise erfassten und vergnüglich eingesetzten Sprachunterschieden der Figuren ausdrückt. (Zur gleichen Zeit hatte in der Sprachwissenschaft die Soziolinguistik mit der Unterscheidung verschiedener sozial determinierter „Codes“ ihre internationale Konjunktur). Weder Lodemann, der sich dezidiert nicht als Genre- oder Serienautor verstand, noch einem anderen Autor gelang in den Folgejahren ein Gegenstück oder überhaupt ein Ruhrgebietskrimi auf dem Niveau der unvergesslichen Anita.

Wir dürfen also trotz dieses vorzeitigen Geniestreichs an der Beobachtung festhalten: Erst Mitte der 1980er Jahre erscheinen vermehrt und gleichzeitig, auch in anderen Regionen, zumeist kurze und wenig komplexe Kriminalromane von nichtprofessionellen Autoren, teils im Selbstverlag, teils in alternativen und ,Nischenverlagen', für die sich die Bezeichnung „Regionalkrimi“" einbürgert. Das hatte und hat immer noch Züge einer grassroots-Literatur, und derartige Texte, oft autodidaktisch oder auch in Hobby-Schreibzirkeln entstanden, gibt es heute zahlreich und flächendeckend in ganz Deutschland, auch wenn sie oft nur in der allerengsten Umgebung ihre nicht sehr zahlreichen, aber lokalpatriotischen Leser und Leserinnen finden und den überregionalen Buchhandel nicht erreichen. Dies ist einerseits eine völlig legitime und vermutlich sehr befriedigende Tätigkeit zwischen Freizeitspaß und Selbstverwirklichung - hat aber auch zum abwertenden Stereotyp des Regional-,,Grimmis“ (so etwa der Kritiker Thomas Wörtche) beigetragen. Noch war auf professioneller und kommerzieller Ebene das branding, also die Marke Regionalkrimi nicht durchgesetzt, aber es war nur eine Frage der Zeit, bis auch etablierte Verlage die dort schlummernden Absatzmöglichkeiten erkennen und systematisch nutzen würden.

\section{Durchbruch in der Eifel}

Die US-Army und der deutsche Regionalkrimi haben vermutlich nur eine Gemeinsamkeit: Der Durchbruch gelingt ihnen, im Abstand von etwa 45 Jahren, in der Eifel. Beim Krimi ist der (nun überregionale!) Erfolg von Jacques Berndorfs Serie von zunächst 13 Titeln zwischen 1989 und 2006 entscheidend, die alle den Landschaftsnamen im Titel tragen: von Eifel-Blues (1989) über Eifel-Schnee (1995), Eifel-Müll (2000) bis Eifel-Kreuz (2006); Gesamtauflage bis dahin etwa drei 
Millionen Exemplare, danach noch zehn weitere Titel. Der investigative Journalist Michael Preute (*1936), so Berndorfs bürgerlicher Name, wählte für seine genrekonform und routiniert erzählten, nun auch deutlich komplexeren und durchaus spannenden Fälle also die bewaldete Gegend zwischen den Bischofssitzen von Köln, Aachen und Trier, die nach verbreitetem Vorurteil von Dauerregen, Schützenvereinen und katholischer Frömmigkeit geprägt ist, aber auch ein weiteres deutsches Spitzenbier und eine letzte US-Air Base aufzuweisen hat. Berndorf kombiniert nun, um mit Roland Barthes zu sprechen, zahlreiche „Realitätseffekte“ en détail mit der eher unwahrscheinlichen Stilisierung des Landstrichs als Brutstätte von Kriminalität en gros. Damit bedient er zwei gegenläufige Erwartungen oder Stimmungen seiner Leserschaft: die Sehnsucht nach einem romantisch verklärten Landleben, wie auch die (nicht ganz falsche, aber doch sehr zugespitzte) Überzeugung, dass hinter der idyllischen Fassade eben so viel Lug und Trug, Mord und Totschlag lauert wie seit Sodom und Gomorra in der bösen Großstadt. Publiziert hat Berndorf die frühe Serie freilich genau dort: beim GrafitVerlag in Dortmund, einem linken Kleinverlag, zu dessen finanzieller Sanierung und weiteren Profilierung als guter Adresse für regionale wie internationale Kriminalliteratur er wesentlich beigetragen hat.

Expandiert hat aber nicht nur Grafit, sondern ein neuer Verlagstypus, der sich über die Marke Regionalkrimi definiert und finanziert. Beispiele sind der Emons-Verlag in Köln (wo ein inzwischen so hoch geschätzter Autor wie Friedrich Ani begonnen hat), der augenzwinkernd mit dem Slogan „Neue deutsche Heimatliteratur“ wirbt; sodann KBV in Hillesheim in der Eifel, dessen Verleger Ralf Kramp nicht nur Berndorfs spätere Werke, sondern auch seine eigene Eifel-KrimiReihe verlegt und nebenbei ein Krimi-Themen-Hotel mit Buchhandlung leitet. Schließlich der Gmeiner Verlag aus Meßkirch in Oberschwaben, der das Geschäftsmodell am konsequentesten durchgesetzt hat: eine große Zahl von einschlägigen Titeln zu verlegen, die primär nach den Handlungsorten sortiert werden, so dass der Verlagsprospekt tatsächlich als dicht besetzte Deutschlandkarte präsentiert werden kann.

Dass dieses Geschäftsmodell „sich rechnet“, lässt sich auch daran ablesen, dass literarische Qualitätsverlage wie Fischer, Suhrkamp, der Deutsche Taschenbuchverlag oder Piper nicht nur dem allgemeinen Boom der Kriminalliteratur (mit Übersetzungen und ambitionierten deutschsprachigen Autoren) Tribut zollen, sondern auch vor Regionalkrimis im engeren Sinne nicht mehr zurückschrecken. Ganz aktuell lässt sich beispielsweise ein ziemlich neues Subgenre mit großem Bestsellerpotential erkennen. 


\section{Das neueste Erfolgsmodell: Der sogenannte Alpenkrimi}

Zuerst einige Namen und Fakten. Erstens: Volker Klüpfel und Michael Kobr (beide *1971), ein Studienrat und ein Journalist, sind laut Spiegel „das erfolgreichste Autorenduo“ Deutschlands. Sie haben den Kommissar Kluftinger („Klufti“) aus ihrer Heimatstadt Kempten im Allgäu erfunden, der sich bisher durch fast zehn Fälle - von Milchgeld (2003) bis Grimmbart (2014) und allerlei private, meist komische Verwicklungen ,gewurschtelt" hat. Nach dem Debüt in einem regionalen Kleinverlag erscheinen ihre Titel inzwischen als Hardcover des Piper Verlags mit Startauflagen bis zu 250000 Exemplaren, sowie als Hörbücher, und sind in den Bestsellerlisten der Nachrichtenmagazine Spiegel und Focus dauerhaft weit oben platziert, ohne es hingegen in die literarisch orientierte KrimiZeit-Bestenliste zu schaffen. Innovativ und charakteristisch ist zweifellos, dass dieses Duo seine neuesten Titel auf Tourneen als Bühnen-Performance präsentiert, mit 80 (!) Terminen in einem Sommer, meist in Bayern, aber auch im ,preußischen Ausland‘: Köln, Hamburg und Berlin. In München haben sie im Circus Krone gastiert, den früher einmal der CSU-Vorsitzende und Ministerpräsident Franz Joseph Strauß gefüllt hat, von anderen Führerfiguren ganz zu schweigen.

Zweitens: Rita Falk (*1964), Polizistengattin aus dem niederbayerischen Landshut, scheute vor der Genrebezeichnung „Provinzkrimi“ schon für ihre beiden ersten kalorienreichen Erzählwerke Winterkartoffelknödel (2010) und Dampfnudelblues (2011) nicht zurück. In denen berichtet „der Eberhofer Franz“, Streifenpolizist in Niederkaltenkirchen, in der Ich-Form und im Präsens von seinen Abenteuern in Beruf und Familie, teils grotesk, teils drollig, stets bei gutem Essen und Trinken und auch mit erotischen Genüssen - von schlank bis mollig - untermischt. Die narrative Konstruktion und der Sprachduktus sind ganz offensichtlich von der pseudomündlich-monologischen Erzählkunst des virtuosen Österreichers Wolf Haas inspiriert, die hier allerdings auf eine sehr plumpe Art heruntergebrochen und ihrer dekonstruktiven Schärfe zugunsten eines dumpfen Populismus beraubt wird. Ein Glossar für niederbayrische Ausdrücke und die Originalrezepte „von der Oma“ sind als paratextuelles Bonusmaterial beigefügt. Beide Paperbacks vom seriösen Deutschen Taschenbuch Verlag hielten sich über viele Monaten in den Bestsellerlisten und werden fortlaufend durch neue krimi-kulinarische Köstlichkeiten ergänzt, zuletzt Zwetschgendatschikomplott (2015) und Leberkäsjunkie (2016).

Auf diesen Listen findet sich, drittens, wenngleich weiter unten, auch Jörg Maurer mit bisher acht „Alpenkrimis“, von Föhnlage (2009) 
bis Schwindelfrei ist nur der Tod (2016). Sein Serienheld, Kommissar Jennerwein, der im Hochgebirge, ja sogar in der Steilwand oberhalb von Garmisch ermittelt, signalisiert den literarischen, genauer: intertextuellen Anspruch schon namentlich: Der „Jennerwein Girgl“ war ja der berühmteste bayrische Wildschütz, 1877 von einem Rivalen im Wald gemeuchelt, noch heute ein Volksmythos. Weiterhin durchziehen zahllose Motti und Zitate, Jodler, Gemsenbildchen, Musiknoten, Rätselund Quizfragen, aber auch Schwärzungen von ,deftigen ${ }^{6}$ oder gar obszönen Ausdrücken den 350-Seiten-Text, dem noch ein Briefwechsel mit den literarischen Helfern folgt. All dies signalisiert: Hier treffen Postmoderne und szenische Virtuosität auf Oberbayern mitsamt seinen Klischees. Tatsächlich war Jörg Maurer Deutschlehrer, Theaterleiter, Rundfunkmoderator und gastiert weiterhin als vor allem regional erfolgreicher Musikkabarettist mit anspruchsvollem Programm.

Der Alpen-Krimi, so könnte man in seinem Sinne kalauern, lebt anders als die Gemsen nicht nur ganz oben - und ein eindeutiges Urteil über ihn ist schwer zu fällen. Auch Grundsätzliches und Historisches muss bedacht werden: Das ehemalige Königreich, der jetzige „Freistaat“ Bayern pflegt (wie auch der Fußballclub, der seinen Namen trägt) bis heute das ausgeprägteste Selbst- und Sonderbewusstsein unter allen deutschen Ländern (bzw. Vereinen). Seine Landschaften und Orte sind seit vielen Generationen beliebte und umsatzstarke Tourismusziele mit außergewöhnlichen Attraktionen (besonders gilt dies für Oberbayern: vom Münchner Hofbräuhaus ganz unten über das Märchenschloss Neuschwanstein bis hinauf zur Zugspitze, Deutschlands höchstem Berg, wo angeblich der Kommissar Jennerwein amtiert). Und die Bayern pflegen mehrheitlich stolz - und ungeachtet ihrer technologischen Avanciertheit - ihre Folklore bis hinab zum Münchner Oktoberfest, die vielen anderen Deutschen bekanntlich exotisch oder skurril vorkommt. Die von Ministerpräsident Strauß einst angestoßene und von Edmund Stoiber, einem seiner Nachfolger, mit dem Slogan „Laptop und Lederhosen" propagierte, fraglos sehr erfolgreiche Modernisierung des alten Agrarlandes und seine Transformation zu einer high-techRegion hat eine hybride Kultur hervorgebracht, die ihrer erschöpfenden Analyse noch harrt.

In den erwähnten Krimis wird sie jedenfalls ganz ins Komische gewendet, daraus resultiert eine Mischform von Krimi und Komödie, um nicht zu sagen Klamauk. Die Geschichten von Rita Falk könnte man ganz leicht szenisch bearbeiten und sofort im „Komödienstadel“ aufführen; diese einst sehr populäre Volkstheater-Fernsehsendung gibt es wohl nicht mehr, aber ins Erste Programm haben es die FalkKrimis auch so geschafft. Das Duo Kobr/Klüpfel und der Kabarettist Maurer haben ihrerseits, wie gesagt, ihren performatic turn zur Bühne längst vollzogen. Auf der Strecke bleibt bei alldem nur allzu leicht der 
Kriminalroman. Das Whodunit ist hier bloß noch ein dünner roter Faden zur Aufreihung von slapstick-Szenen, und von weiterer Zerfaserung bedroht. Entertainment und event-Kultur verschlingen das literarische Genre. Oder: Der Kriminalroman wird zur universalen Erzählform, indem er sich auflöst. Zum Glück gibt es Alternativen.

\section{Mehr oder weniger gelungen}

An Jacques Berndorfs Eifel-Klassikern hat man kritisiert, dass sie durch Austausch der Ortsnamen auch in andere Regionen verlegt werden könnten. Dem könnte man zwar mit guten Gründen widersprechen - aber: Der entscheidende Punkt, wenn man sich denn für Kriminalromane als Literatur interessiert, ist zumindest angesprochen. Gerade weil regionale Elemente auf sehr verschiedenen funktionalen und literarischen Ebenen verwendet werden, ist es für die genrespezifische Qualität eines Krimis entscheidend, ob und wie solche Regionalismen, besonders wenn sie im Roman hervorgehoben werden, für den Gattungskern, also für den Fall oder die Ermittlung etwas hergeben, mit ihnen plausibel verknüpft sind, sie unverwechselbar machen - oder ob sie bloße Staffage, Hintergrund oder Füllmasse bilden. Man müsste also, um ein berühmtes Aperçu von Walter Benjamin zu missbrauchen, sagen können: „Auf diesem Sofa, und nur auf diesem Sofa kann die Tante ermordet werden!“

Damit stellt sich konsequenterweise die Frage, welche Regionen oder welche Art von Regionen welche Leserbedürfnisse befriedigen. Das Wiedererkennen vertrauter Orte ist ein hübscher Effekt, bleibt aber trotz rasant gestiegener Mobilität der Leserschaft begrenzt. Die Vorfreude auf das nächste Ferienziel mag bei mancher Kaufentscheidung eine Rolle spielen und wird von den Verlagen entsprechend stimuliert - etwa auch, um über die Grenzen zu schauen, mit Provence- oder BretagneKrimis von französischen, britischen oder deutschen Autoren: ein transnationaler Regionalismus! Am wichtigsten scheint aber doch, zumindest für die schon erwähnten innerdeutschen Regionen, dass sie neben markanten Stereotypen (die Eifel: katholisch und verregnet, aber mit gutem Bier) genügend Raum für Projektionen lassen, die Autor und Leserschaft dann ausmalen können - plausibel oder auch nicht. Nützlich scheint es dabei, wenn der oder die Autor/in bei aller Vertrautheit mit der Region von außen kommen und zu einem ,doppelten Blick' fähig sind: Berndorf stammt aus Osnabrück, Wolf aus dem Ruhrgebiet, die derzeitige "Quoten-Queen“ Nele Neuhaus mit ihren Taunus-Krimis kommt aus Paderborn.

Mit solchen Erwägungen drücken wir uns aber immer noch vor der Gretchenfrage: Wie geht der regionalistische Blick mit literarischer Qualität zusammen? (Dass es gehen muss, zeigen schließlich Regionalromane wie Buddenbrooks oder Ulysses schon seit rund hundert 
Jahren.) Aber bleiben wir in unserer, der „Regionalliga“ (wie es bezeichnenderweise auch im Fußball heißt). Was wir hier Regionalität nennen, ohne es ganz genau definieren zu können: also äußere Gegebenheiten, Traditionen und Mentalitäten, müsste den Krimiteig jedenfalls durchsäuern wie die Hefe oder aufgehen lassen wie das Backpulver. Ein positives, wenn auch nicht besonders komplexes Beispiel finden wir in einem Krimi des schon erwähnten Routiniers Felix Huby (bürgerlich Eberhard Hungerbühler, *1936) mit dem Titel Bienzle und das Narrenspiel (1988). Schauplatz ist die fiktive Kleinstadt Venningen, hinter der das für seinen „Narrensprung“ berühmte Rottweil in Württemberg jedoch leicht zu erkennen ist, wo eine weltweit erfolgreiche mittelständische Industrie ebenso blüht wie das regionale Traditionsbewusstein. Ausgerechnet hier kommt es während der alemannisch-schwäbischen „Fasnet“ (dem hier seit Jahrhunderten auf alte Weise gefeierten Karneval) zu einem Mord, den nun der Stuttgarter Kommissar Bienzle aufklären muss, der doch eigentlich nur den folkloristischen „Narrensprung“, also die Parade der schweren Holzmasken genießen wollte. Für die Dramaturgie des Romans wie auch der sehenswerten Tatort-Adaption von 1994 (die nach Protesten in Rottweil allerdings in Ravensburg gedreht werden musste!) bietet dies die Chance, unschuldig Verdächtige wie auch den tatsächlichen Täter bis zum finalen Showdown unter den archaischen Masken zu verstecken. Gleichzeitig werden die Konflikte innerhalb des weltweit erfolgreichen, insofern ,typisch schwäbischen' high-tech-Familienbetriebs offensichtlich - vielleicht ein Krisensymptom in Zeiten beginnender Globalisierung?

Ein Gegenbeispiel bietet die bereits erwähne Nele Neuhaus (bürgerlich Cornelia Löwenberg, *1967) mit ihrer Serie von bisher sieben Bänden und einer Gesamtauflage von etwa fünf Millionen Exemplaren. Besonders beliebt war wohl Schneewittchen muss sterben (2010); zuletzt erschien Die Lebenden und die Toten (2014). Alle Bände spielen, wie der Untertitel bzw. die Serienkennung Ein Taunus-Krimi sagt, im bergigen Hinterland von Frankfurt am Main, nach wie vor der deutschen, wenn nicht sogar europäischen Finanz- und Luftverkehrsmetropole. Treffend gezeichnet ist diese Region, mit exklusiven Kleinstädten wie Bad Homburg oder Kronberg, Wohnorten der wohlhabenden Frankfurter Prominenz, und weiteren Orten, wo sich neben den Villen und modernen Bürogebäuden noch alte Dorfkerne und eine bodenständige, meist am hessischen Dialekt (gelegentlich auch in einer serbokroatisch getönten oder sonst hybriden Variante) erkennbare Urbevölkerung hält - was immer wieder zu netten, teils komischen ,interkulturellen Szenen und Effekten führt. Charakteristisch für Neuhaus ist aber eine eher lockere Verbindung zwischen diesem Setting und den jeweiligen Kriminalfällen, die eben auch ,woanders' vorgefallen sein könnten. Zugleich ist die Autorin sichtlich bemüht, möglichst viele verschie- 
dene und möglichst aktuelle soziale, politische, ökologische, und vor allem zwischenmenschliche Konflikte und Probleme im Rahmen der Kriminalgeschichte und ihres regionalen Rahmens einzubinden und ,abzuhandeln', auch wenn sie mit dem Fall an sich nichts oder nur wenig $\mathrm{zu}$ tun haben. Der ist lediglich noch ein narratives Transportband für Zeitgeistthemen, die dann gern mit einem erklärenden oder didaktischen Unterton dargelegt werden: Frau Neuhaus war und ist zugleich eine beliebte Autorin von Pferde- und Mädchenbüchern. Ihre TaunusKrimis finden - dennoch oder deswegen? - ein außerordentlich breites Leserinteresse, treffen aber - besonders in den texttreu verfilmten Fassungen des Zweiten Deutschen Fernsehens - auch regelmäßig auf scharfe und spöttisch formulierte Ablehnung, beispielsweise in den fundierten und meinungsbildenden Fernsehkritiken der Frankfurter Allgemeinen Zeitung.

\section{Closing the Gap? Neue Rahmenbedingungen für den deutschen Krimi}

Neben dem Lokal- und Regionalkrimi von nicht- und halbprofessionellen Verfassern und den eben vorgestellten Bestsellern gibt es aber drittens eine beträchtliche Gruppe von Autoren und Autorinnen, für deren Kriminalromane der Begriff ,Regionalkrimi“ entschieden zu eng und auch völlig verfehlt wäre, obgleich oder vielmehr weil sie regional oder lokal differenzierte Schauplätze nicht nur als Handlungsrahmen, sondern als topographische, soziale, historisch-kulturelle Handlungsräume mit erkennbarer „Eigenlogik“ nutzen. Von ihnen wird abschließend noch die Rede sein; zuvor sind jedoch einige Informationen und Überlegungen $\mathrm{zu}$ Veränderungen im deutschen Literaturbetrieb, speziell im Sektor der Kriminalliteratur, nötig und hilfreich, die für sie und ihre Bücher besonders relevant sind.

Den Begriff von der „Eigenlogik der Städte“ hat die Literaturwissenschaftlerin Julika Griem aus der Urbanistik übernommen und auf Kriminalromane, -filme und Fernsehserien übertragen (zunächst bezeichnender Weise wieder am Beispiel Tatort) ${ }^{5}$. Um einen Raum in der erwähnten Vielschichtigkeit ,sprechend“ zu machen, oder gar, nach der schönen Formulierung des Historikers Karl Schlögel, ,im Raum die Zeit lesen“ zu können, ist allerdings ein sehr viel weiteres Repertoire an narrativen Formen und Strategien erforderlich, als es die Pioniere des Neuen deutschen Kriminalromans zur Verfügung hatten. Die jetzt angesprochenen Autoren und Autorinnen, mit wenigen

5. - Vgl. Julika Griem, Sebastian Scholz (Hrsg.), Tatort Stadt. Mediale Topographien eines Fernsehklassikers, Frankfurt am Main, Campus Verlag, 2010, S. $20 \mathrm{ff}$. 
Ausnahmen aus einer mittleren Generation, haben bereits an einem Prozess partizipiert und von ihm profitiert, den ich - unter Bezug auf eine ähnliche Beobachtung von Pierre Bourdieu - an anderer Stelle „,nachholende Modernisierung“ der Kriminalliteratur genannt habe ${ }^{6}$. Dies bezieht sich darauf, dass die Kriminalliteratur zwar seit ihren Anfängen Mitte und Ende des 19. Jahrhunderts als eine Literatur der gesellschaftlichen Moderne verstanden wurde, zugleich aber aufgrund ihrer früh kodifizierten narrativen Spielregeln auf die technischen und damit darstellerischen Errungenschaften verzichtete, welche die avantgardistische oder ,modernistische“ Erzählliteratur seit 1900, verstärkt aber nach 1920, entwickelt hat.

So steht der Kriminalroman (wie auch der Spionage- oder Agentenroman) für eine moderne Literatur, die sich vormoderner Techniken bedient, für „modernity without modernism“, wie man in Anlehnung an eine Überlegung von Peter von Matt sagen darf ${ }^{7}$. Dies gilt international, auch im anglophonen Bereich - man vergleiche in den 1920er Jahren nur die etwa zeitgleich publizierten Romane von Virginia Woolf und der jungen Agatha Christie. In Deutschland hat sich die Adaption modernistischer Erzähltechniken und -formen, zum Beispiel des nichtlinearen oder polyperspektivischen Erzählens, der stream-of-consciousness-Technik, einer forcierten Intertextualität und Selbstreferenzialität, der Hybridisierung von Genres usw. durch die historischen Umstände gleich mehrfach verzögert. Eher mühsam wurde sie in den 1950er und 1960er Jahren in der mainstream-Literatur, auch von den namhaften Autoren der Gruppe 47, erarbeitet; im ohnehin rückständigen deutschsprachigen Krimi erst eine Generationsspanne später.

Dabei spielten neben dem inzwischen erreichten state of the art der internationalen, besonders der angloamerikanischen, westeuropäischen oder skandinavischen Kriminalliteratur auch Anregungen durch weltweit erfolgreiche, mehr oder weniger postmoderne „Beinahe-Krimis“ (so Sigrid Thielking) wie Der Name der Rose von Umberto Eco (1980), Paul Austers New York Trilogy (1985/86) oder Patrick Süskinds Parfum (1985) ein wichtige Rolle ${ }^{8}$. Dies liegt nun aber bereits wieder um eine

6. - Jochen Vogt, Ist der Deutsche Schäferhund des Inneren Monologs fähig? Nachholende Modernisierung des Erzählens im gegenwärtigen deutschen Kriminalroman. Vortrag beim Deutschen Germanistentag, Kiel, 2013 (ungedruckt).

7. - Vgl. Pierre Bourdieu, «Le marché des biens symboliques », in: L'année sociologique 22 (1971), S. 49-126. Gekürzte englische Fassung in: Poetics (Amsterdam), 14 (1985), H.1/2, S. 13-44. Jochen Vogt, ,,Modern? Vormodern? Oder Postmodern? Zur Poetik des Kriminalromans und seinem Ort im literarischen Feld“, in: Véronique Liard (Hrsg.), Verbrechen und Gesellschaft im Spiegel von Literatur und Kunst, München, Peter Lang, 2010, S. 23; vgl. Peter von Matt, Die Intrige. Theorie und Praxis der Hinterlist, München, Hanser, 2006, S. 453-465.

8. - Sigrid Thielking/Jochen Vogt (Hrsg.), „Beinahekrimis“-Beinahe Krimis?, 
Generationsspanne zurück, und im Blick auf die heute produktive Autorengeneration darf man durchaus sagen, dass sie den deutschsprachigen Kriminalroman inzwischen auch im internationalen Vergleich konkurrenzfähig gemacht hat. Und dies, ohne spezifisch deutsche (oder österreichische oder deutschschweizerische) Aspekte - also auch Regionalismen - einem globalisierten Standard- oder Bestsellermodell zu opfern. Die grundsätzliche Frage, die hinter diesem Adaptionsprozess modernistischer Schreibweisen im Kriminalroman steht, lautet ganz analog zu der aus Anlass des „Regionalismus“ diskutierten: Kommen sie der Spezifik des Genres, der Erwartung der Leserschaft an einen Kriminalroman, also der „Spannung“ zwischen Fall und Aufklärung zugute, bereichern, erneuern und differenzieren sie das Genre - oder überschreiten und sprengen sie seine Grenzen und führen zu neuen Themen, Formen und zu neuen Positionen im literarischen Feld. Dies bleibt vorerst eine offene Frage, die an unterschiedlichen Beispielen gründlich zu diskutieren wäre.

Im Rückblick darf man allerdings sagen: Die genannten Anregungen und Impulse waren ihrerseits Symptome und zugleich Verstärker einer ganz allmählichen Überwindung oder doch Überbrückung des legendären Gap zwischen Hochkultur und Massenkultur, den Leslie Fiedler 1969 beschworen hatte ${ }^{9}$. Es ist bekannt, dass diese Kluft in der deutschen Literatur besonders tief und nachhaltig ausfiel und sich bis zur Literaturpolitik der Weimarer Klassik zurückverfolgen lässt. Hier soll exemplarisch aber nur das Literatursystem in der Bundesrepublik betrachtet werden. Eine kategorische Geringschätzung und massive, teilweise geradezu bösartige Abwertung des Kriminalgenres durch die normsetzenden Instanzen des literarischen Systems, wie den schulischen Deutschunterricht, die akademische Literaturwissenschaft, die Literaturkritik in der sogenannten Qualitätspresse, die Vergabe von Preisen durch Akademien und andere Gremien, nicht zuletzt durch die Praxis der öffentlichen Bibliotheken und die Polemik der Bibliothekarsverbände lässt sich bis in die 1950er und 1960er Jahre breit dokumentieren und hat sich zaghaft erst seit den 1970er Jahren aufgelockert.

Man kann allerdings fragen, ob die pauschale Abwertung und Ausgrenzung, oder zumindest das demonstrative Desinteresse an diesem Schrifttum auf der Kehrseite nicht einen umso größeren Freiraum für ein ,wildes ' Lesen, für individuelle Vorlieben und Geschmacksurteile schafft, die ein rein kommerzieller, nur durch den Markt und die Werbemedien vermittelte Zugang zu dieser Art Literatur

Bielefeld, Aisthesis, 2014, darin besonders die Einleitung von Sigrid Thielking, S. 7-19.

9. - Leslie Fiedler, Cross the Border, Close the Gap (1969), jetzt in: L. F., A New Fiedler Reader, Amherst N.Y., Prometheus Books, 1999, S. 270-294. 
ohne weitere Umstände und Mittlerinstanzen befriedigen kann. Für meinen Lieblingskrimi (oder Autor) muss ich mich nicht rechtfertigen, er ist einfach mein Favorit!

Nun zeigt sich jedoch, wie schon gesagt, seit den 1970er Jahren eine anfangs noch zaghaft zu nennende Verschiebung. Auch jene normativen Instanzen betreiben inzwischen eine vorsichtige Revision der harten Ausgrenzung von Kriminalliteratur. Öffentliche Bibliotheken fänden ohne Spannungsliteratur noch weniger Zuspruch als ohnehin. Im Unterricht der Schulen finden Krimiformate - vom Krimi-Hörspiel für Kinder bis zur Pflichtlektüre der Oberstufe - inzwischen einen Raum; regional akzentuierte Krimis sind besonders beliebt; oder sie werden im Rahmen des kreativen Schreibens auch von einem Literaturkurs selbst verfasst. Ähnlich ist die Situation in den Literaturwissenschaften; einschlägige Lehrveranstaltungen sind möglich und üblich, in der Anglistik und Amerikanistik, oder auch der Romanistik öfters, in der Germanistik seltener. Kriminalliteratur bleibt dort immer noch ein gern belächeltes Steckenpferd einige Liebhaber; als Forschungsgegenstand dürfte sie nach wie vor karriereschädigend wirken. Zumindest unterliegt sie einer doppelseitigen Ausgrenzung: den Literaturwissenschaftlern ist sie zu soziologisch, den Kulturwissenschaftlern zu literarisch. Auch der vielzitierte und modische cultural turn der Literaturwissenschaft spart - jedenfalls in Deutschland - Massenliteratur und damit den Krimi weithin aus und erprobt den neuen methodischen Zugriff lieber an kanonischen Gegenständen der Hochkultur.

Vermutlich ist all dies aber gar nicht so wichtig, weil insgesamt die Steuerung des Medien- und Literaturkonsums durch die klassischen Bildungsinstitutionen gegenüber der Macht der Medien und der Werbung immer mehr ins Hintertreffen gerät. Eine Schlüsselrolle dürfte im Blick auf unser Thema nach wie vor das Fernsehen spielen, wo Krimiformate in verschiedenen Längen (von 30 bis 90 Minuten) und Serien seit den 1980er Jahren zum quantitativ dominierenden fiktionalen Sendungstyp aufgestiegen sind ${ }^{10}$. Dies gilt im sogenannten „dualen System“ des deutschen Fernsehens sowohl für die öffentlichrechtlichen wie für die privaten Sender und umfasst einheimische Produktionen in verschiedenen Formaten, aber auch US-amerikanische Standardserien wie Law and Order oder CSI, sowie Qualitätsserien und Mehrteiler (serials) insbesondere aus Großbritannien und den skandinavischen Ländern. Das durchschnittliche Wochenprogramm auch der ,Öffentlich-Rechtlichen' ist heutzutage mit einem dichten Netz von Fernsehkrimis überzogen, vom Vorabendprogramm (gern mit Krimikomödien) über die prime time (klassischer Sendeplatz für Tatort,

10. - Vgl. Reinhold Viehoff, „,Der Krimi im Fernsehen. Überlegungen zur Genreund Programmgeschichte“, in: Jochen Vogt, Medien Morde, a.a.O., S. 89-110. 
seine spin-offs und Konkurrenzserien) bis zum späten Abend (bevorzugter Wiederholungstermin für Tatorte in den Regionalprogrammen und für beliebte Importserien). Selbst Festtage wie Weihnachten, Ostern und Pfingsten oder andere kirchliche bzw. gesetzliche Feiertage, die früher durch Gesetzeskraft frei von sex and crime gehalten wurden, sind inzwischen dicht mit Krimis besetzt.

Aber auch die Informationsmedien haben der Kriminalliteratur, dem Kriminalfilm und den TV-Programmen zunehmend Platz eingeräumt. Das weitaus auflagenstärkste Massenblatt, die Bild-Zeitung greift gern kontroverse Tatort-Folgen auf und leuchtet auch das Privatleben der Serienstars aus. Seriöse Regionalzeitungen wie der Tagesspiegel aus Berlin, die Westdeutsche Allgemeine Zeitung in Essen oder die Stuttgarter Zeitung sind zumeist dicht am Interesse und an den Vorlieben breiter Leserschichten orientiert und berichten über Neuerscheinungen, TV-Programme, prominente Autoren und Schauspieler sowie Lesungen und „Krimi-Events“ aller Art. Und selbst die deutschlandweit gelesenen ,nationalen' Tageszeitungen, also die Frankfurter Allgemeine Zeitung, Die Welt und die Süddeutsche Zeitung, oder auch Wochenzeitungen wie die große Zeit und der kleine, politisch links profilierte Freitag haben seit einigen Jahren monatliche Themenseiten oder auch Beilagen, sogenannte „Specials“ zur Kriminalliteratur eingeführt. Die Zeit publiziert überdies monatlich eine „Krimi-Bestenliste“, die von zwanzig kompetenten Kritikern und Kritikerinnen erstellt und vom Moderator Tobias Gohlis leserfreundlich kommentiert wird ${ }^{11}$.

All dies sind Anzeichen einer allmählichen Aufwertung spannender Literatur oder auch nur der Anpassung an das Kauf- und Leseverhalten breiter Schichten. Im Buchhandel und in den Werbemedien stehen ja seit längerem die dicken Hardcover-Krimis deutscher, vor allem aber auch internationaler Autoren neben den ,hochliterarischen' Romanen und dürfen jetzt auch ebenso viel kosten - zumeist zwischen 19 und 29 Euro. Es scheint jedenfalls, dass zumindest im Urteil, im Lesegeschmack und im Kaufverhalten dieses Publikums der Gap zwischen ,hoher und ,niederer" Literatur, zwischen E (für Ernsthaftigkeit) und U (für Unterhaltung) wenn nicht überbrückt, so doch sehr viel schmaler geworden ist als ehedem. Ungeachtet dieser erfreulichen Veränderungen zeigt sich aber auch, dass der überwiegende Sachverstand in Sachen Krimi nicht institutionell, insbesondere nicht akademisch organisiert ist, was immer schon für das Genre typisch war (man denke nur an die gigantisch-skurrile Sherlock-Holmes-Forschung der Amateurexperten), aber auch für Liebhaberkulturen insgesamt charakteristisch ist. Im Zeitalter der Digitalisierung kann das nur heißen, dass dieses Expertenwissen

11. - www.zeit.de/krimizeit-bestenliste 
und eine kritische Meinungsvielfalt überwiegend im Internet zu suchen ist, wo man ohne große Mühe die einschlägigen Portale, Archive und Diskussionsforen finden kann ${ }^{12}$.

\section{Breite und Vielfalt neuester deutscher Kriminalliteratur}

Wenn ich nun zum Schluss dieser Bestandsaufnahme - und als Einladung zur Lektüre - einige jener gegenwärtig produktiven Autoren und Autorinnen vorstelle, die Regionalismus und Modernismus auf jeweils individuelle Weise und mit verschiedenen Intentionen verbinden und damit den deutschsprachigen Kriminalroman auf ein höheres, auch international konkurrenzfähiges literarisches Niveau gehoben haben, will ich sehr deutlich den subjektiven Charakter meiner Auswahl betonen, auch wenn sie durch die Literaturkritik und die Vergabe zahlreicher Literaturpreise weithin gestützt wird. Kehren wir auf einer kurzen Rundfahrt, welche die „Eigenlogik“ verschiedener Städte oder regionaler Schauplätze und deren Relevanz für eine literarisch anspruchsvolle Kriminalliteratur zumindest andeutet, zunächst ins Ruhrgebiet zurück.

Die Kernregion des bevölkerungsreichsten Bundeslandes NordrheinWestfalen bewältigt seit Mitte der 1960er Jahre mehr oder weniger erfolgreich die Transformation und Integration einer schwerindustriell - von ,Kohle und Stahl ${ }^{\circ}$ - geprägten Region in die postindustrielle Dienstleistungsgesellschaft und ist insofern mit bestimmten Gegenden in Frankreich, Großbritannien oder den USA zu vergleichen. Prägend war im Zuge der Industrialisierung seit den 1870er Jahren eine starke Einwanderung, zunächst von polnischen Arbeiterfamilien, die zu gelebter Multikulturalität, alltäglicher Solidarität und Toleranz beitrug und die Mentalität eines pragmatischen Realismus förderte, dem zufolge der „,Ruhrmensch sacht wat Sache is“. All diese Züge findet man mühelos in den bislang zwölf Romanen von Jörg Juretzka (*1965), von Prickel (1998) bis Trailer Park (2015), die zumeist an der Ruhr spielen und den Gelegenheitsdetektiv (oder Hausmeister, oder Barkeeper) Kristof Kryszinski (polnischer Familienname!), genannt „Krüschel“, zum Helden haben. Typisch ist freilich, dass Juretzka die eher problematischen Aspekte der regionalen Entwicklung betont, die prekären Existenzen versammelt, die randständigen Orte oder "Nicht-Orte“ wie Kneipen, Spielhallen, Autofriedhöfe, Schrott- und Campingplätze übereinander türmt und ineinander schiebt, also ,verdichtet', und die -

12. - Zum Einstieg: www.bokas.de (= Thomas Przybilkas Krimi-Tipp. Sekundärliteratur, bisher 61 Folgen); www.krimi-couch (= kommerziell orientiert, aber informativ); www.culturmag.de/crimemag (= unabhängig, aktuell, kompetent und meinungsfreudig); www.kaliber38.de (desgleichen); www.krimilexikon.de (= H. P. Karr: Lexikon der deutschen Krimi-Autoren. Online-Version). 
realiter weit verbreitete - halbproletarische Spießigkeit oder den neureichen Protz nur als Kontrastfolie verwendet. Ganz ähnlich geht er mit der Sprache um, die er seinem ,Maulhelden' und dessen Kumpanen oder Rivalen in den Mund legt: Das „Ruhrdeutsche“ - kein alter deutscher Dialekt, sondern neuartiger Typ einer großstädtischen Umgangssprache - forciert und überzeichnet er so sehr, dass es sich quasi selbst dekonstruiert und eine teils handfeste, teils geradezu surrealistische Komik erzeugt. Vergleiche mit amerikanischen Krimi-Autoren wie Kinky Friedmann oder Carl Hiaasen sind durchaus angebracht. Wenn ein Kritiker gesteht, seine Juretzka-Lektüre sei von zahllosen „Lachsalven“ begleitet, so ist nur hinzuzufügen, dass die komische Wirkung, weil sie überwiegend auf „Ruhrdeutsch“ als Sprach- und Erfahrungsform beruht, ohne eine gewisse Vertrautheit damit schon bald auf regionale Verstehensgrenzen stößt.

Weder solche Probleme noch lautes Gelächter wird es bei den Romanen von Norbert Horst $\left({ }^{* 1956)}\right.$ geben, die zunächst (seit Leichensache, 2004) im angrenzenden, gern als provinziell verspotteten Ostwestfalen spielen, teils aber auch (bis Mädchenware, 2014), in Dortmund, der zehntgrößten deutschen Stadt (inzwischen auch mit eigener Tatort-Serie), und die in der Tradition des (amerikanischen) Polizeiromans das realistische Kontrastprogramm zu Juretzka liefern. „Realismus“ heißt hier detailgenaue und treffsichere Schilderung der Umwelt, besonders aber der Abläufe und Probleme ,authentischer" Ermittlungen, was sich zwanglos aus dem jahrzehntelangen Hauptberuf des Autors als Kriminalkommissar in Bielefeld erklärt. „Realismus“ heißt in diesem Fall aber auch eine sehr bewusste Arbeit an der Erzählsprache, die sich teils multiperspektivisch, reportagenah und in einer Art Telegrammstil als „Präsens-Roman“13 darstellt; teils etwas konventioneller, aber psychologisch tiefschürfend an die Erlebnisperspektive der Ermittlerfigur gebunden ist.

Die Ermittler an der Ruhr, im Ballungsraum und im Netz der Schnellstraßen, sind natürlich meist mit dem Auto unterwegs, bei Horst mit den Dienstwagen der Polizei, Juretzkas „Krüschel“ mit allem, was noch vier Räder hat. Hingegen erreicht Hauptkommissar Robert Marthaler von der Mordkommission in Frankfurt am Main seine Einsatzorte zumeist mit dem Fahrrad, was insofern überrascht, als die Stadt nicht besonders radlerfreundlich und teilweise auch hügelig ist wie das Umland. Andererseits kommt er, besonders in der verstopften Innenstadt, damit oft schneller ans Ziel als mit dem Wagen. Bekannt ist aber auch, dass sein Schöpfer Jan Seghers (bürgerlich Matthias

13. - Armen Avanessian, Anke Hennig (Hrsg.), Der Präsensroman, Berlin, De Gruyter, 2013. 
Altenburg *1958) ein geradezu fanatischer Radfahrer ist und seine Erlebnisse auch diaristisch festhält ${ }^{14}$.

Im erzählerischen Zugriff bewähren sich Marthalers Fahrten, indem sie diese Großstadt und ihr dicht besiedeltes Umland, den „Ballungsraum Rhein-Main“, topographisch sehr genau, ja empirisch überprüfbar erschließen, aber auch - in der Nachfolge von früh verstorbenen Autoren wie Jörg Fauser ( $\$ 1987)$ oder Jakob Arjouni $(† 2013)$ - die starken Kontraste des städtischen Lebens in den Blick rücken. Frankfurt ist als Standort zahlreicher Großbanken (beginnend mit der Europäischen Zentralbank) nicht nur internationales Finanzzentrum, sondern mit dem Rhein-Main-Flughafen auch ein Drehkreuz des weltweiten Flugverkehrs, und zumindest unter diesen Aspekten immer noch die einzige global city in Deutschland. Zugleich ist der Anteil hier lebenden Ausländer und Immigranten seit Jahrzehnten der höchste in Deutschland, was keinen Gegensatz zu der in vielen Stadtteilen noch spürbaren Bodenständigkeit der Einheimischen bedeuten muss, sondern seit langem zu einer multiethnischen, vielfarbigen und lebendigen Mischung beiträgt. Aber auch die überdurchschnittlich hohe Kriminalitätsrate (zu der wiederum der Flughafen erheblich beiträgt), sollte nicht verschwiegen werden.

Seghers zeigt diese Vielschichtigkeit mit ihren Kontrasten oder Konflikten in bislang fünf Romanen (von Ein allzu schönes Mädchen, 2006, bis Die Sterntaler-Verschwörung, 2014) und versucht auch die unheilvollen Auswirkungen zeithistorischer Ereignisse einzubinden. In Partitur des Todes (2008) spielt die Verfolgung der Frankfurter Juden durch die Nationalsozialisten eine wichtige Rolle oder: Die Rosenholz-Akte (2009) spielt indirekt auf einen niemals aufgeklärten Prostituiertenmord in der Stadt an, der in der frühen Bundesrepublik zum legendären Skandal wurde.

Weiter nach Süden, jenseits des sogenannten „Weißwurst-Äquatora“: Friedrich Ani (*1959) wird derzeit von vielen Kritikern als wichtigster deutscher Kriminalschriftsteller angesehen; eine treue Leserschaft hat er mit seiner knapp zwanzigbändigen Serie um den Kriminalkommissar Tabor Süden (von Die Erfindung des Abschieds, 1998, bis M. Ein Tabor-Süden-Roman, 2013) gewonnen. Der klärt mit seinen Kollegen keine Mordfälle, sondern „Vermissungen“ in München auf. Die Abweichung vom üblichen Krimi-Schema reduziert den Stellenwert von Gewaltkriminalität und technisch-medizinischen Ermittlungen und lenkt Südens Blick aufs Psychologische und in die Vergangenheit, wo meist die individuellen, familiären und sozialen Ursachen für das Verschwinden einer Person liegen. In vielen dieser Fälle gibt es kein

14. - Matthias Altenburg, Jan Seghers' Tagebuch mit Toten, Reinbek, Rowohlt Verlag, 2006. - Zwerst als Blog unter www.janseghers.de. 
Verbrechen, oft werden die Vermissten gefunden, auch wenn sie vielleicht nicht zurückkehren. Eigene Beschädigungen haben Südens Intuition geschärft, seine Empathie vertieft ${ }^{15}$. Insgesamt stehen diese relativ konventionell erzählten Kurzromane am ehesten in der Tradition eines Georges Simenon oder auch des Schweizers Friedrich Glauser.

Wie aber steht es um das Regionale? Selten nur führen die Ermittlungen Süden über die Münchner Stadtgrenzen hinaus. Aber auf seine Weise behandelt er die bayerische Hauptstadt als Region - und zwar in scharfem Kontrast zu dem, was Medien und Politik gern als deren Glanz- und Selbstbild verbreiten. Denn Anis „München leuchtet“ eben nicht - seine Serie ist eine einzige Widerlegung des Wortes von Thomas Mann, mit dem sich die reiche und kulturgesättigte Stadt immer noch gern schmückt, dieser bevorzugte Wohnsitz einer fröhlichen leisure class (neudeutsch: ,Schickeria', münchnerisch: ,BussiBussi-Gesellschaft'), zugleich die deutsche Großstadt mit den höchsten Lebenshaltungskosten. Mit Süden ziehen wir, seiner alternativen „Eigenlogik“ folgend und oft zu Fuß, durch diejenigen Stadtteile, in denen kleinbürgerliche oder verarmte Menschen leben, teils noch in traditionellen Lebensformen, teils bindungs-, halt- und trostlos. Es lohnt sich insofern, diese Romane mit einem Stadtplan zu lesen; und auch hier spielen die transitären „Nicht-Orte“, in erster Linie die Restaurants, Gasthäuser, Bierschwemmen und Eckkneipen eine wichtige Rolle - als eine Art Sicherheitsnetz für den Vermisstensucher, der in seiner eigenen Stadt auf paradoxe Weise zugleich beheimatet und verloren ist.

Wenn Anis Romane einen partiellen Stadtplan ergeben, so geht der Österreicher Wolf Haas (*1960) aufs Ganze: aufs ganze benachbarte Österreich. Seine Serie um den ehemaligen Polizisten, Krankenwagenfahrer, Chauffeur, Privat- und Gelegenheitsdetektiv Simon Brenner hat längst Kultcharakter und wird - eine einmalige Ausnahme - vom Lesepublikum, den Krimispezialisten, aber auch der mainstream-Literaturkritik mit Lob überschüttet. Das liegt sicher nicht daran, dass Haas ,den Brenner“ in acht Romanen (von Auferstehung der Toten, 1996, bis Brennerova, 2014) durch verschieden Wiener Bezirke, nach Salzburg, Zell am See und Kitzbühel (also zu touristischen hot spots führt) oder auch in den 17. Grazer Bezirk, Brenners Geburtsort Puntigam, ,wo das gute Bier gebraut wird“. Es liegt vielmehr ganz eindeutig an der Erzähl- bzw. Kunstsprache, die der promovierte Linguist Wolf Haas für diese Romane oder genauer: für den anonym bleibenden Erzähler erfunden hat, der in einer permanenten Stammtischrunde das

15. - Thomas Kniesche, ,Der ,Kommissar für die, die weg sind'. Friedrich Anis Tabor-Süden-Romane und die Topographie des Traumas", in: andererseits. Yearbook of Transatlantic German Studies 3 (2013), S. 125-145. Auch online: http://andererseits. library.duke.edu/ 
Wort zu führen scheint. Es ist eine pseudo-mündliche, zwischen Unsinn und Tiefsinn schwankende, hochgradig performative, intertextuelle und selbstreferenzielle Redeweise, die auch überregional verständlich bleibt, weil sie relativ sparsam mit Austriazismen umgeht, sich zugleich aber auf eine hochliterarische Tradition von Sprachskepsis und Sprachspiel stützen kann, die von Karl Kraus bis zu Thomas Bernhard, Ernst Jandl und Elfriede Jelinek reicht - also auf einen posthabsburgischen Sonderweg des Modernismus.

Wo Haas in die Ferne schweift, führt Alfred Komarek (*1945) uns mit bislang sechs Romanen (beginnend 1998 mit Polt muss weinen) und einem Geschichtenband räumlich und geologisch in die Tiefe der „Preßhäuser“ im niederösterreichischen „Weinviertel“ (das sind die in den fruchtbaren Lößboden gegrabenen Kelter- und Lagerräume der dörflichen Winzer) und psychisch, sozial und moralisch in die menschlichen Abgründe, die sich überall auftun, wo man nur tief genug gräbt. Dies tut der Gendarmerie-Inspektor Simon Polt, bald auch schon im Ruhestand, der im fiktiven Burgheim und dem Wiesbachtal meist per Fahrrad unterwegs ist. Seine Gemütlichkeit darf man allerdings ebenso wenig verkennen wie die ganz und gar nichtmodernistische Erzählweise, die ein wenig altväterlich an Dorfgeschichten des 19. Jahrhunderts erinnert, zuletzt freilich (Alt, aber Polt, 2015) allzu betulich klingt.

Konventionell muten auch - wir überqueren eine weitere Staatsgrenze - die bislang neun Kriminalromane von Hansjörg Schneider (*1938) an (von Silberkiesel, 1993, bis Hunkelers Geheimnis, 2015), in denen der Kommissär Hunkeler die Ermittlungen führt, zuletzt ebenfalls im Ruhestand. Er lebt und wirkt in der traditionsreichen, ein wenig selbstgerechten und dank der chemischen Großindustrie weiterhin prosperierenden Grenzstadt Basel, womit wir wieder in der dreistaatlichen „Regio“ am Oberrhein angekommen sind. Die staatlichen Grenzen zwischen der Schweiz, Frankreich und Deutschland, regional zwischen dem Doppelkanton Basel, dem Elsass und der badischen Schwarzwaldregion, sind offiziell durchlässig, bürokratisch und mental aber doch wieder nicht. Die Grenze(n) und die Grenzüberschreitung, die historischen Zäsuren und regionalen Kontraste sind Leitmotive in diesen teils elegischen, teils satirischen Geschichten (mit Seitenhieben etwa auf das Schweizer Bankenwesen), die mit feiner Ironie erzählt sind, auch was den alternden Hunkeler selbst angeht, als Kriminalfälle jedoch eher unkompliziert und versöhnlich erscheinen. Mit ihnen reiht sich Schneider umstandslos in die Tradition seiner Landsleute Glauser und Dürrenmatt ein, die schon seit den 1930er bzw. 1950er Jahren auch mit innerschweizerischen Regionalismen gearbeitet haben ${ }^{16}$.

16. - Vgl. Jochen Vogt, „Krimis, Antikrimis, ,Gedanken'-Krimis. Wie Friedrich Dürrenmatt sich in ein gering geschätztes Genre einschrieb“, in: Véronique Liard, Marion 
Und nun in den Schwarzwald, auf den Hunkeler über den Rhein hinweg oftmals schaut, und den umliegenden deutschen Südwesten. Uta-Maria Heim ist dort 1963 geboren, ihre ersten Kriminalromane wurden schon Anfang der 1990er Jahre ausgezeichnet; inzwischen sind es um die zwanzig, wobei die Grenzen zu zehn ,nicht-K-Romanen' flieBend bleiben. Von Das Rattenprinzip (1991) bis zu Wem sonst als Dir. (2014) bleiben jedenfalls zwei Charakterzüge konstant: eine ,schräg'anarchische Grundhaltung der Autorin, die es liebt, politische, gesellschaftliche und literarische Konventionen gegen den Strich zu bürsten, und ein Repertoire an literarischen Kenntnissen und Strategien, mit dem sie die meisten Kollegen überragt. Das letztgenannte Buch (mit einem berühmten Hölderlin-Zitat als Titel) macht dies besonders deutlich ${ }^{17}$. Heim schreibt vielstimmig, multiperspektivisch, intertextuell, mischt fiktionales Erzählen mit historischen oder fingierten Dokumenten, lässt Monologe oder Bewusstseinsströme einen ganzen Roman tragen. Stärker als andere Autoren, von den Verbalartisten Haas und Juretzka oder den Alpenjodlern einmal abgesehen, verwendet sie Dialekteinsprengsel, sehr pointiert und nuanciert im linguistisch wie psychologisch sensiblen Sprachdreieck von Schwäbisch, Alemannisch und Badisch. Aber sie pflegt auch eine Tiefendimension des Regionalismus: Von den Höhen der Schwäbischen Alb, wo 1940 in der berüchtigten Anstalt Grafeneck zehntausend psychisch Kranke vom Nazistaat ermordet wurden, geht es in Wem sonst als Dir. hinab nach Hölderlins Tübingen, in die Landeshauptstadt Stuttgart und in die Abgründe deutscher Vergangenheit, durch die Gespenster des Terrorismus der 1970er Jahre ebenso geistern wie Holocaust und Weltkrieg. Und im Krimirahmen wird ein zwanzig Jahre altes Fehlurteil zumindest mental revidiert und der Mordfall findet seine unverhoffte Lösung.

Ein letzter Blick geht aber noch einmal eher in die Weite als in die Tiefe. Einige, meist jüngere Autorinnen und Autoren arbeiten in einer Richtung, die man Entgrenzung des Regionalen nennen könnte. Damit meine ich nicht so sehr Veit Heinichen, der als deutscher Schriftsteller mit ausgeprägt kultur- und zeithistorischem oder auch pädagogischem

George (Hrsg.), Dürrenmatt und die Weltliteratur - Dürrenmatt in der Weltliteratur, München, Peter Lang, 2011, S.215-236; weiterhin die Handbucheinträge über Friedrich Glauser (,Matto regiert“, „Wachtmeister Studer“) in: Marion Bönnighausen, Jochen Vogt (Hrsg.), Literatur für die Schule, Paderborn, Fink UTB, 2014, S. 242-244.

17. - „Wem sonst als Dir.“ lautete die Widmung, die der unglückliche Dichter seiner Lebens- und Leidensliebe Susette Gontard ins Geschenkexemplar seines Romans Hyperion schrieb. - Zur literarischen Komplexität von Heims Roman vgl. Jochen Vogt, „Verriegelte Vergangenheit. Bodenlose Gegenwart. Über Tempusgebrauch, Redeformen, Regionalismus, Intertextualität und Sonstiges in zwei Kriminal-(?)-Romanen von UtaMaria Heim“, in: Tanja van Hoorn (Hrsg.), Zeit, Stillstellung und Geschichte im deutschsprachigen Gegenwartsroman, Hannover, Wehrhahn Verlag, 2016, S. 75-93. 
Ehrgeiz eine Art Regionalkrimi seiner italienischen Wahlheimat Triest und ihrer historischen Wandlungen entwickelt hat (8 Bände, von Gib jedem seinen eigenen Tod, 2001, bis Im eigenen Schatten, 2013), sondern vielmehr ein Erzählmodell, das in Anlehnung an die jüngste deutsche Zeitgeschichte Nähe und Ferne, Gegenwart und Vergangenheit, Regionalität und Globalisierung dialektisch aufeinander bezieht.

Ulrich Ritzel (*1940), langjährig erfahrener Polizei- und Gerichtsreporter „,in Ulm, um Ulm und um Ulm herum“ (ein beliebter deutscher ,Zungenbrecher"!) hatte seit 1999 zunächst acht Krimis verfasst, die eben dort spielen und als "soziale Enquête“ deutscher Gegenwartsgesellschaft ${ }^{18}$ zu lesen sind, mehr oder weniger tief aber auch in einer deutschen Vergangenheit wurzeln, die sich als ,untot ${ }^{*}$ erweist, insofern Nazi-Verbrechen verschiedener Art unheilvoll bis ins Heute wirken und verdrängte Schuld erneut aufbricht. Für den zweiten und den letzten Roman aus dieser Gruppe, Schwemmholz (2000) und Beifang (2009), erhielt Ritzel jeweils den Deutschen Krimipreis des Folgejahres. Zwei Romane, die nach der Übersiedlung des Autors (und seines Ermittlers Berndorf, jetzt Kommissar im Ruhestand) in die neue deutsche Hauptstadt entstanden, wählen verschiedene Perspektiven auf die Metropole; da geht es um den langen Schatten der Balkankriege aus den 1990er Jahren, die auf Berlin als Exil vieler Kroaten fallen (Schlangenkopf, 2011), oder auch um die hausgemachte Korruption von Politik und Big Business (Trotzkis Narr, 2013). In beiden Fällen unterliegt dem aber ein Grundthema, das man literarisch bis zu Döblin oder Fritz Lang zurückführen könnte: der Moloch Metropolis und seine Opfer, denen hier das spürbare Mitgefühl des Autors gilt.

Ähnlich wie bei Ritzel verlief die bisherige Karriere des deutlich jüngeren Oliver Bottini (*1965), der sich seit 2005 mit seinen Romanen um die Freiburger Kriminalpolizistin Louise Bonì einen Namen gemacht hat (zuletzt Im weißen Kreis, 2015), inzwischen aber in Berlin lebt. In Der kalte Traum (2013) geht es um die schwäbische Provinz (wieder einmal Rottweil!) und um die deutsche Hauptstadt, aber auch um Serbien, wo in diesem Fall das nachwirkende Unheil im Krieg begann. Deutlicher noch als Ritzel ist mit dieser zeithistorisch plausiblen Verknüpfung von Tatund Handlungsorten ein tendenzieller Genrewechsel vom Polizeiroman zum Agententhriller verbunden, der sich in Bottinis nächstem Buch Ein paar Tage Licht (2014) in der Konstellation Berlin-Algerien und mit dem Thema ,Arabischer Frühling oder islamischer Terror?` eindrucksvoll fortsetzt.

18. - Vgl. Stefanie Abt, Soziale Enquête im Kriminalroman. Am Beispiel von Henning Mankell, Ulrich Ritzel und Pieke Biermann, Wiesbaden, Deutscher Universitätsverlag, 2004. 
Auch Merle Kröger (*1967) nutzt, in Anlehnung an authentische Ereignisse, in Grenzfall (2013) einen regionalen Tatort, die deutschpolnische Staatsgrenze in Mecklenburg-Vorpommern, wo sich dann Probleme entladen, die andernorts - in diesem Fall in Rumänien - ihren Ursprung haben. In ihrem letzten Buch Havarie (2014), das thematisch die Flüchtlingsströme des Jahres 2014 von Nordafrika nach Südeuropa aufgreift, geht sie noch weiter und wählt ein luxuriöses Kreuzfahrtschiff auf dem Mittelmeer als Schnittpunkt so vielfach sich überkreuzender Lebensschicksale und Konfliktlinien, wie sie erst die globalisierte Welt hervorbringt. Dem entspricht eine Schnitt-Technik (short cuts), die von der auch als Filmemacherin erfahrenen Autorin themenadäquat und wirkungsvoll in Szene gesetzt wird.

Schließlich wäre Zoe Beck (*1975) zu erwähnen, deren letzte beiden Kriminalromane Brixton Hill (2014) und Schwarzblende (2015) überhaupt nicht mehr in Deutschland, sondern in London spielen. Natürlich ist dies nicht das düster-neblige London, das deutsche Krimis in den 1950er Jahren in später Edgar-Wallace-Nachfolge gern beschworen, auch nicht die Heimat von Sherlock Holmes oder Jack the Ripper, sondern das heutige Epizentrum globaler Vernetzung und Bedrohung, wo globale Finanzströme, unbewältigte ethnische Vielfalt und sonstige innenpolitische Probleme, die virtuelle Welt und der islamische Terrorismus eine gefährliche Mischung abgeben. In Becks Büchern kommt es dann tatsächlich zu kaum noch beherrschbaren Explosionen der Gewalt.

Ob die Kriminalliteratur sich grundsätzlich und dauerhaft als fähig erweisen wird, derartige Entwicklungen und Zustände literarisch zu fassen - und wie sie sich dafür inhaltlich und strukturell verändern müsste - wage ich nicht zu entscheiden. Ihren Weg zu einem Universalgenre des 21. Jahrhunderts zu verfolgen, dürfte aber spannend werden, mindestens so sehr wie ein außergewöhnlich guter Krimi.

\section{Nachfrage: Und was ist mit Berlin?}

Dies ist eine naheliegende Frage, die einem besonders in Amerika gern gestellt wird. Schnippisch könnte man antworten: Berlin ist nicht Deutschland. Oder weniger polemisch: Die größte deutsche Stadt, jetzt auch wieder Hauptstadt eines vereinten Deutschland, ist nicht nur was den Krimi angeht, sondern in vielfacher Hinsicht: ökonomisch, politisch, sozial und atmosphärisch ein Sonderfall, vielleicht sogar ein Problemfall. Ihr Status als Metropole ist im kollektiven Bewusstsein der Deutschen keineswegs so fraglos verwurzelt wie etwa der von Paris im französischen. Soweit ich sehe - und ohne die Ansätze bei Ulrich Ritzel und anderen abzuwerten - hat bislang noch kein deutscher Kriminalroman die Komplexität und Widersprüchlichkeit dieser 
Stadt überzeugend erfasst, die ja allen Kriterien einer Region entspricht, sich selbst aber allzu oft als Metropole (miss)versteht. Außer Frage steht, dass kaum eine Region oder Stadt in Deutschland so sehr von den tiefgreifenden Zäsuren der Zeitgeschichte (1945, 1949, 1961, 1989) geprägt (und teilweise beschädigt) wurde wie die einstige und jetzige Hauptstadt; aber auch, dass sie sich gegenwärtig in einem dynamischen Veränderungsprozess befindet, der sie als boomtown für deutsche und internationale Touristen, für junge Menschen aus aller Welt und für die sogenannte Kreativindustrie (Kunst, Neue Medien, Mode und Design, Entertainment) attraktiv gemacht hat. Andererseits ist sie ökonomisch nach wie vor nicht aus eigener Kraft lebensfähig, krankt an einer chronisch provinziellen Innenpolitik, erheblichen sozialen Reibungen und Konflikten, nicht zuletzt in Fragen der Integration von Migranten. All dies nun literarisch zu verarbeiten, nicht nur im Krimi, ist eine Aufgabe, die leicht gestellt und schwer zu erfüllen ist - zumal sie unvermeidlich in den Schlagschatten von Alfred Döblins Jahrhundertroman Berlin Alexanderplatz aus dem Jahr 1929 gerät. Der populäre Frankfurter Dichter Robert Gernhardt hat Ende der 1990er Jahre, während eines längeren Aufenthalts an der Spree, die von der Literaturkritik oft erhobene Forderung nach einem repräsentativen Berlin-Roman ins Satirische gewendet: ,den Hauptstadtroman, den schreib ich euch nicht / wenn es hoch kommt, dann pack ich das Hauptstadtgedicht" 19 .

Natürlich gibt es inzwischen auch nicht wenige Berlin-Krimis, aber noch keiner scheint der verzwickten ,Eigenlogik“ dieser Stadt so recht auf die Schliche gekommen zu sein ${ }^{20}$. Möglicherweise geht dies vorerst am ehesten im Rückgriff auf die historische Dimension, was dann zu interessanten Varianten des Krimischemas bzw. zu hybriden Formen führt. Wo die Gegenwart (noch) nicht recht zu fassen ist, könnte man in die Vergangenheit oder gar in die Zukunft ausweichen. Dies tun jedenfalls zwei Erzählwerke, die ich abschließend empfehlen möchte. Das eine sind die bislang fünf Bände (von Der nasse Fisch, 2007, bis Märzgefallene, 2015) des Kölner Autors Volker Kutscher, der seine Fälle in den historisch genauestens recherchierten Untergang der Weimarer Republik einpasst und nun in den ersten Wochen der Naziherrschaft angekommen ist. Eine lesenswerte Typuskombination von KriminalGroßstadt- und Geschichtsroman, deren einzige Schwäche man darin

19. - Robert Gernhardt, Couplet vom Hauptstadtroman, in: Ders.: Gesammelte Gedichte 1954-2004, Frankfurt am Main, S. Fischer, 2005, S. 674f.

20. - Das gilt auch für die durchaus lesenswerten und mit dem Deutschen Krimipreis ausgezeichneten Romane von Elisabeth Herrmann. Charakteristisch scheint mir, dass etwa in Das Dorf der Mörder (2013) die Darstellung des unter massiven NachWende-Problemen leidenden brandenburgischen Umlandes im Atmosphärischen wie im Analytischen sehr viel eindrücklicher wird als die der aufblühenden Metropole. 
sehen kann, dass der Autor den Drang in die Breite nicht energisch genug zügelt. Das andere ist der Roman Plan D von Simon Urban, der im September 2011 erschien und im Oktober desselben Jahres spielt, und zwar in der Hauptstadt der ,wiederbelebten“ DDR, also eine Art von kontrafaktischer Geschichtsschreibung, oder ein Kriminal-undZukunftsroman. Das gibt, abgesehen von einer wirklich spannenden Krimi- oder eigentlich Agentenhandlung, Gelegenheit zur ebenso fundamentalen wie witzigen, manchmal ins Groteske überdrehten Abrechnung mit dem alten System, besonders der „Staatssicherheit“, und gleichzeitig, da Urban über erzählerische Phantasie und einen Sprachwitz verfügt wie einst der junge Günter Grass, zu einem ganz eigenwilligen Lesevergnügen. 
